\title{
Wearable Organic Optoelectronic Sensors for Medicine
}

\author{
Ashu K. Bansal, Shuoben Hou, Olena Kulyk, Eric M. Bowman, and Ifor D. W. Samuel**
}

Non-invasive sensors are desirable for a wide range of medical measurements and especially for ubiquitous monitoring of a patient's health. Medical measurements are typically taken infrequently during a visit to a physician or hospital. However, continuous monitoring (for example, the monitoring of blood pressure $)^{[1]}$ is preferable as it gives a much more complete picture. Wearable sensors are therefore desired for unobtrusive long-term continuous physiological and health monitoring which is crucial for many chronic illnesses, neurological disorders, depression, drug addiction, and rehabilitation. ${ }^{[2,3]}$ Furthermore, such sensors are useful for a wide range of medical measurements as they play a crucial role in human-machine interactions, ${ }^{[4]}$ in vitro diagnostics ${ }^{[5]}$ and as epidermal electronics systems. ${ }^{[6]}$ Ideally, wearable sensors should be lightweight, compact, flexible, non-invasive, simple to make, and low cost. These requirements place numerous simultaneous demands on the sensors. Recently, a wearable pressure sensor using gold nanowires was reported to monitor the blood pulse in real-time, ${ }^{[7]}$ and Samsung has launched a commercial product in the form of wrist watch to monitor heart rate and blood pressure. In another interesting study, Rogers and coworkers have reported a compact wearable surface electromyography (sEMG) sensor based on silicon electronics to make an epidermal electronics system. ${ }^{[6,8]}$

The sensors described above have great potential but here we demonstrate a complementary approach using organic semiconductors to make flexible wearable optoelectronic sensors that can be made by solution-processing techniques. Organic semiconductor devices have attractive properties of tunable light emission, simple fabrication on flexible substrates with scope for miniaturization, and the ability both to generate and detect light. ${ }^{[9-11]}$ The potential of organic optical devices in medicine, both as sensors and manipulators of biological activity, has only begun to be explored recently for point of care diagnosis, ${ }^{[12]}$ as a lab-on-chip spectrometer, ${ }^{[13]}$ for biosensors, ${ }^{[14]}$ and flexible e-skin. ${ }^{[15]}$ There are also a few reports of animal studies involving implanted organic electronic devices, for example,

\footnotetext{
Dr. A. K. Bansal, S. Hou, O. Kulyk, Prof. I. D. W. Samuel Organic Semiconductor Centre, SUPA

School of Physics \& Astronomy

University of St Andrews

St Andrews KY16 9SS, UK

E-mail: idws@st-andrews.ac.uk

Dr. E. M. Bowman

School of Psychology \& Neuroscience

University of St Andrews

St Andrews KY16 9JP, UK

This is an open access article under the terms of the Creative Commons Attribution License, which permits use, distribution and reproduction in any medium, provided the original work is properly cited.

The copyright line for this article was changed on 12 Dec 2014 after original online publication.
}

DOI: 10.1002/adma.201403560 neuronal photostimulation, ${ }^{[16]}$ microelectrode arrays ${ }^{[10]}$ and in vivo measurement of brain activity, ${ }^{[17]}$ and a report of using an organic light-emitting diodes (OLEDs) light source for photodynamic therapy of skin cancer ${ }^{[18]}$ in humans.

In this communication, we demonstrate that OLEDs and organic photodiodes (OPDs) can be combined to make compact, non-invasive, flexible sensors for medical applications by simple solution-processing methods, and we describe two examples of such sensors. In the first example, we have developed a thin, flexible, light weight, and potentially low-cost muscle contraction sensor which can measure signals from intact muscles to control the movement of active prosthetic devices, such as artificial limbs. ${ }^{[19,20]}$ Moreover, we have demonstrated the feasibility of this all-organic, optoelectronic sensor by controlling a robotic arm so that it mimicked the motion of the arm of a healthy volunteer for two types of muscle contractions. Specifically, we have shown that the sensor can detect both isotonic and isometric types of muscle contraction and distinguish between them. The clear signal obtained means that movement of a volunteer's arm can be used to control a robot which copies the volunteer's movement. We have also performed optical modeling using Monte Carlo simulations to study the effect of anisotropic properties of muscle fibers on the incident light. In the second example, we have made a flexible organic optoelectronic bandage to measure tissue oxygenation and demonstrate it by measuring a healthy adult during blood pressure cuff-induced ischemia. It has important implications in the field of neuroimaging, ${ }^{[21]}$ exercise medicine, ${ }^{[22]}$ and for understanding of the vascular conditions such as peripheral arterial disease. ${ }^{[23]}$ This research demonstrates that the combination of OLEDs and OPDs processed on flexible substrates can open an era of low-cost disposable non-invasive optical sensors for medicine and sports.

The optical muscle contraction sensor we designed works by measuring the amount of incident light backscattered from skeletal muscle tissue. Myosin protein in the sarcomeres of muscles has liquid crystalline properties and is responsible for the anisotropic behavior of muscles. ${ }^{[24]}$ When muscle contracts actin fibers slide along myosin, and the muscle fiber becomes short and wide, thereby scattering incident light anisotropically: light traveling parallel to muscle fibers is scattered differently from light perpendicular to fibers and this anisotropy can be detected by a surface-mounted light source and detectors positioned along and perpendicular to muscle fiber. ${ }^{[19,25]}$ There are several considerations in designing an organic optoelectronic probe (wavelength, geometry, choice of materials, and fabrication). Figure 1a shows a schematic of the sensor mounted on an arm as a flexible bandage, which consists of four photodiodes and a light source in the middle. The photodiodes were prepared using polymer thieno[3,4-b]thiophene/benzodithiophene (PTB7) with (6,6)-Phenyl C71 butyric acid methyl ester $\left(\mathrm{PC}_{70} \mathrm{BM}\right)$ as the active layer. The polymer PTB7 is an interesting low band gap material used in photovoltaics with power 


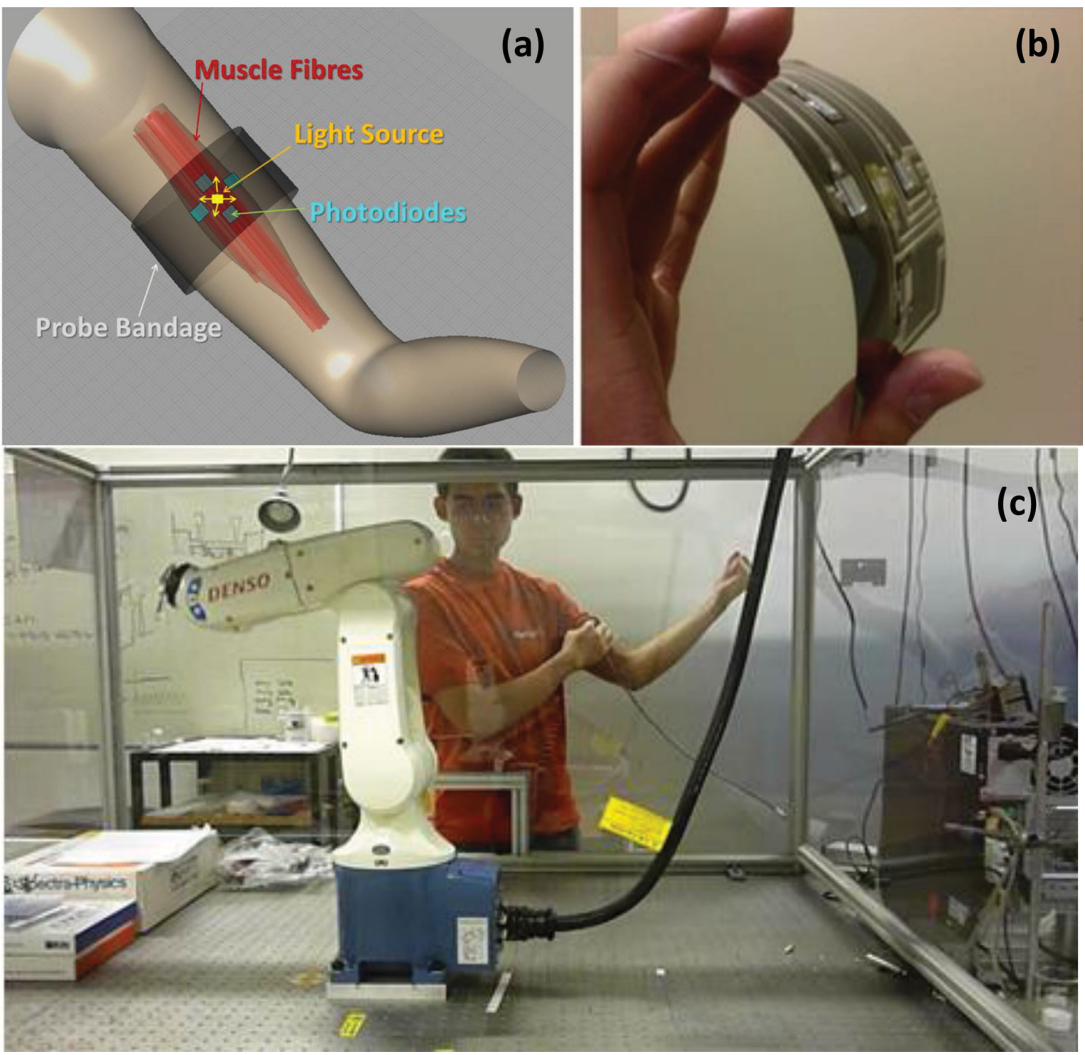

Figure 1. a) Schematic principle of muscle contraction sensor showing an arm containing muscle fibers, and the probe bandage consisting of a source and four photodiodes on top of the arm. b) Photograph of the thin flexible organic optoelectronic sensor. c) Image file of the video in which a robotic arm mimics the movement of the arm of a volunteer.

conversion efficiency up to $9 \% .{ }^{[11,26]}$ The OLED source was prepared using the conjugated polymer Superyellow (SY) as it has broad emission spectrum from 520 to $700 \mathrm{~nm} .^{[27]}$ The properties of the photodiode and SY OLED on plastic substrates are shown in Figure S1(Supporting Information).

For the incorporation of such polymer materials in the sensor two important parameters were taken into account. The first was the selection of an appropriate wavelength and the second was the geometry of the probe. When light interacts with tissue there is both absorption and scattering and thus only light with wavelengths ranging from 600 to $1000 \mathrm{~nm}$, can penetrate into tissue for millimeters to centimeters and return to detectors at the surface. ${ }^{[28]}$ We have chosen the wavelength range 610-700 nm for our muscle contraction sensor based on source and detector properties. The second key parameter in designing the optical probe is its geometry. Skeletal muscle is an inhomogeneous medium in which photons are scattered many times as they travel from the light source to the detector. In this scenario the optical path length is much greater than the physical spacing between the light source and exit points because light is scattered multiple times before it emerges. ${ }^{[29]}$ The further apart the source and detector are, the lower the signal to noise ratio will be, so to have optimum signal to noise we have chosen two photodiodes in each direction to record the signal. Figure $1 \mathrm{~b}$ shows the photograph of the actual sensor having a flexible source in the middle with four flexible photodiodes on a flexible base. Figure 1c shows a picture of the volunteer having the sensor on his biceps and the robotic arm in the foreground.

To guide the design of the sensor, we modeled the light propagation between the source and the detector based on Monte Carlo simulations. Optical modeling was done on a system consisting of a source and detector with a layer of fat, skin, and bulk muscle tissue and results are shown in Figure S2 (Supporting Information). The results show that the further the detector is from the source, the more likely it will record photons backscattered from the muscle. However, this comes at a cost, since the amount of light reaching the detector decreases as the separation increases as shown in Figure S3 (Supporting Information) that shows the optical power recorded by the photodiodes at different distances. Therefore, by taking into account the effect of absorption and anisotropic scattering by fat, skin, and muscle tissue, the model supports the idea that $20 \mathrm{~mm}$ is a suitable distance between the source and the detector to discriminate effectively between the two transverse directions of the scattering. Similar modeling studies have been done on the influence of adipose tissue on muscle oxygenation and found that light can penetrate a layer of adipose tissue if the source and detector are $20 \mathrm{~mm}$ apart. ${ }^{[30]}$ Thus, in designing our sensor we have chosen four photodiodes with spacing of $20 \mathrm{~mm}$ between source and detectors to balance depth sensitivity and signal to noise ratio in our measurements.

This sensor was tested on a 25 -year-old male volunteer who gave informed consent. It was placed over his biceps muscle and held in place by a cloth bandage whilst taking care not to occlude blood circulation. The volunteer then performed a series of isotonic (i.e., the muscle shortens, at a constant exerted force) contractions by either lifting weights or lifting his lower arm and hand. He also performed isometric (i.e., the muscle length remains constant) contractions by either pushing the wall or twisting his arm. ${ }^{[19]}$ The sensor detected the anisotropic change in scattering due to contraction with intrinsic rejection to non-anisotropic signals, such as those resulting from movement of hand. Typical signals detected at the oscilloscope are shown in Figure 2a for isometric and Figure 2b for isotonic contractions. The results show that the signals corresponding to contraction can be easily distinguished from the steady state baseline. The shape of the signals corresponding to isotonic and isometric contractions is clearly different. In particular, in the case of isometric contractions, there is an increase in signal first and then it decreases. In contrast, in the first phase of isotonic contractions, the signal decreases and then it rises. Hence, the sign of the signal is informative about the type of the contraction as shown in Figure 2c, which consists of filtered output from both type of contractions. By increasing the time interval step, the measurement bandwidth was increased, and this process filter out the blood depletion and refilling signals. 
(a)

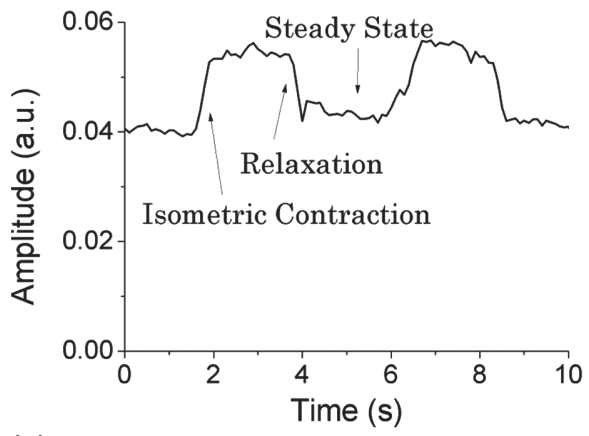

(b)

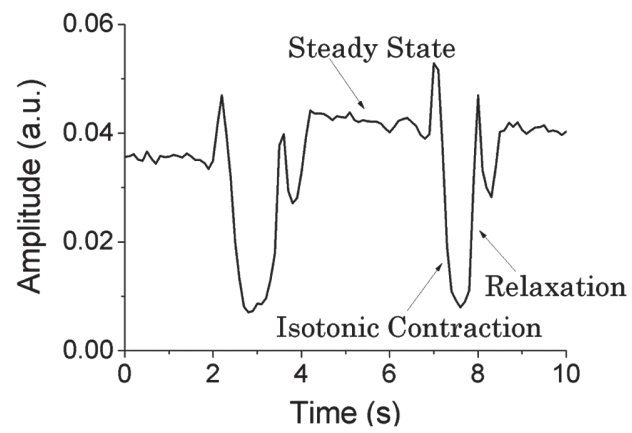

(c)

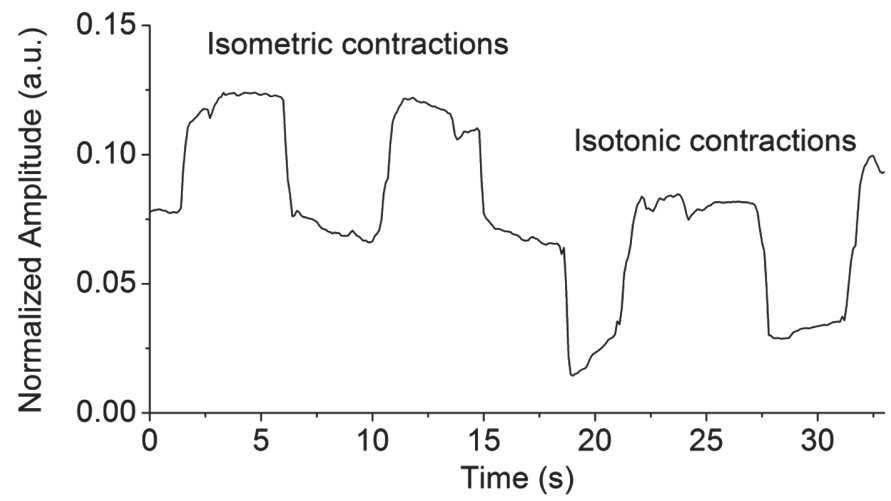

Figure 2. a) Graph showing the signal corresponding to isometric and b) isotonic contractions of a volunteer. c) Measurement showing two isometric contractions followed by two isotonic contractions.

The reason for the contrasting signs of the isotonic and isometric contractions is that during isometric contraction the muscle fibers are constrained to a quasi-constant length. Therefore, differential scattering contributes to the first part of the signal only, and then hematic depletion dominates, leading to an increase of the signal. ${ }^{[19,25]}$ Conversely, in the first part of the isotonic contractions, the muscle is rapidly depleted of blood, and then the differential optical scattering contribution due to the shortening of the muscle fibers occurs.

We checked the feasibility of using our sensor by controlling a robotic arm based on the signal measured from a volunteer. The signal from the sensor was used to control the robot arm, which imitated both isotonic and isometric muscle contractions of the volunteer. The sensor was successfully tested on both the biceps and the deltoid muscles. The videos for both types of muscle contractions are given in Supporting Information. In Video S1, the sensor is placed on the biceps of the volunteer. He then performs isotonic contractions in which he bends his arm as though he were lifting a dumbbell. In this scenario the change in muscle length is mirrored by the movements of the robotic arm. The volunteer then twists his arm and voluntarily generates an isometric contraction, thereby controlling the robotic arm using a second type of muscle contraction. In Video S2, the sensor is placed on the deltoid muscle of the volunteer and similar actions were performed as described above, and the robotic arm copied those actions for isotonic and isometric contractions. There is a small time-lag between the action of the volunteer and the robotic arm in the videos which is due to a threshold value of the signal from the photodiodes being set in the program that controls the robotic arm. Movement of the robotic arm begins once the signal exceeds this threshold value. The clear control of the robot arm shows that our muscle contraction sensor using organic semiconductors could be used to actuate prosthetic devices such as artificial limbs.

In comparison to our sensor, EMG sensors are not able to distinguish such isotonic and isometric contraction which result in limited functionality of active prosthetics based on detection and mimicking isotonic contraction, such as the ability to only mimic flexion and extension of the limbs. In order to mimic resistance to pressure (pushing a door, resistance during gaits, etc.) detection and mimicking isometric contraction is needed. There have been a few attempts to distinguish such contraction such as by analyzing frequency and amplitude of recorded sEMG signals, ${ }^{[31]}$ by learning the patterns of electroencephalogram (EEG) signals generated by the brain, ${ }^{[32]}$ or by optimizing mechanical movement of active prosthetics. ${ }^{[33]}$ Our sensor can distinguish both types of contractions and can add extra functionality to active prosthetics enabling natural movements of the limbs.

The second wearable sensor that we developed is for tissue oxygenation. It is based on near-infrared spectroscopy (NIRS), which is widely recognized as a powerful non-invasive technique to study tissue oxygenation and perfusion. ${ }^{[29,34]}$ This technique is based on the monitoring of blood oxygenation and its kinetics and utilizes the optical properties of endogenous chromophores such as hemoglobin $(\mathrm{Hb})$ and cytochrome $\mathrm{aa}_{3}$ oxidase. Our sensor can measure the changes in the concentrations of oxygenated $\left(\mathrm{HbO}_{2}\right)$ and deoxygenated $\mathrm{Hb}$ and can 


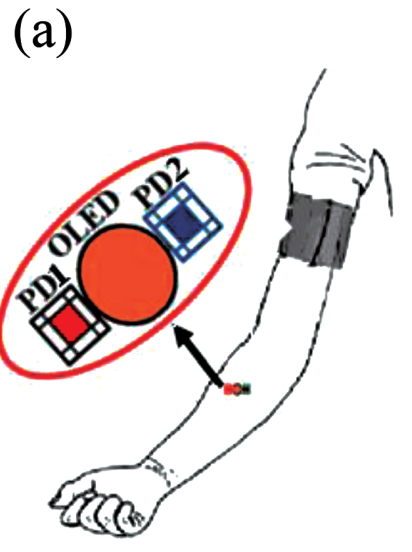

(c) (b)

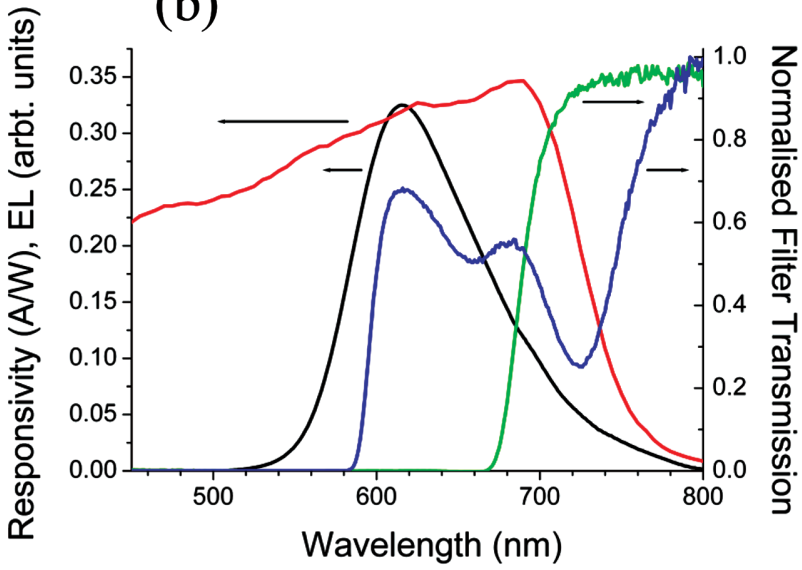

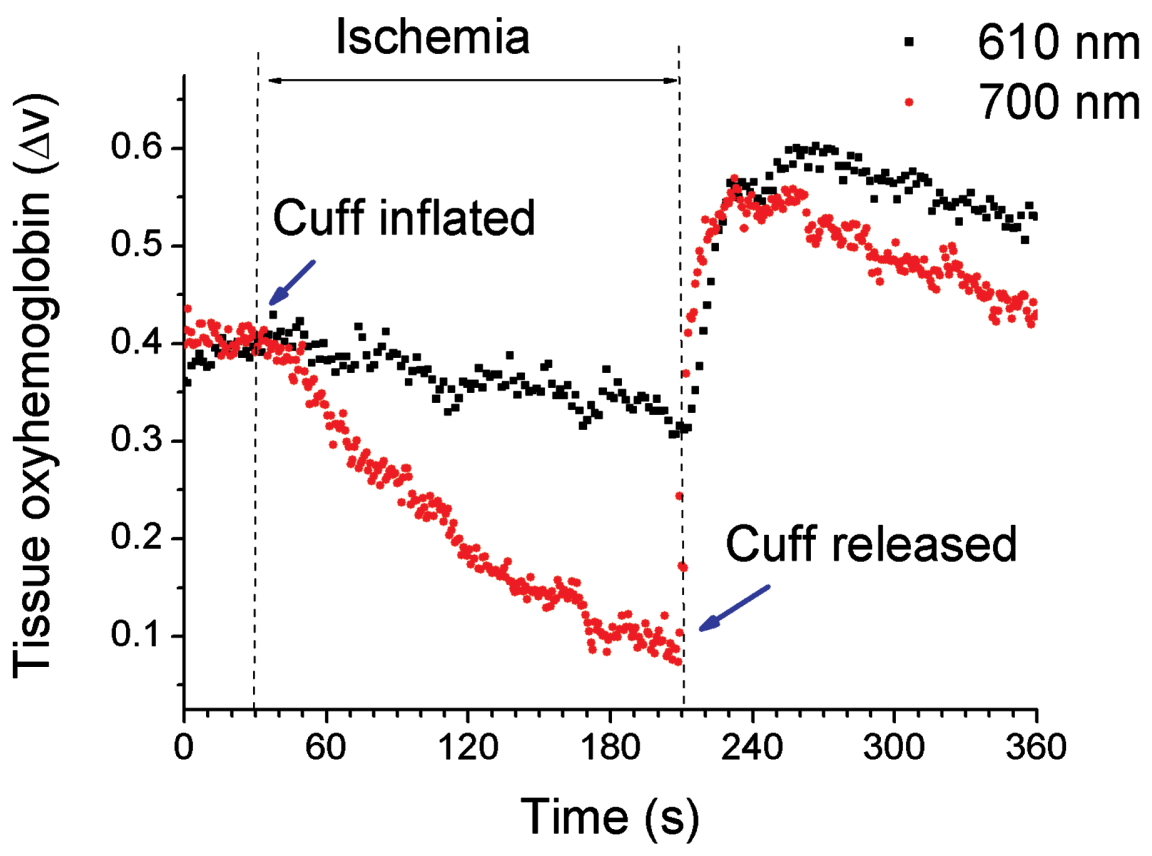

Figure 3. a) Schematic of the optical probe consisting of an OLED source and two photodiodes PD1 and PD2 on a flexible base together with forearm ischemia setup showing blood pressure cuff at arm. b) EL spectra of $\mathrm{OC}_{1} \mathrm{C}_{10}$-PPV OLED (black line) and responsivity of PTB7/PC 70 BM bulk-heterojunction photodiode (red line) on the left axis. The right axis shows the filter transmissions used to select different wavelengths $610 \mathrm{~nm}$ (blue line) and $700 \mathrm{~nm}$ (green line). c) Optical responses at $610 \mathrm{~nm}$ (filled black squares) and at $700 \mathrm{~nm}$ (filled red circles) from forearm skeletal muscle of a subject during $3 \mathrm{~min}$ of blood pressure cuff-induced ischemia and subsequent recovery which is indicative of change of tissue oxygenation at different wavelengths.

monitor the change in cerebral blood volume with high temporal resolution. In designing the sensor we have again considered the appropriate wavelength and geometry. ${ }^{[29]}$ We have chosen two different wavelength bands to show the importance of the appropriate light source. As shown in Figure S5 (Supporting Information), the extinction coefficient of $\mathrm{Hb}$ and $\mathrm{HbO}_{2}$ is high below $600 \mathrm{~nm}$ so we have used 610 and $700 \mathrm{~nm}$ as two detection wavelengths in the sensor. The source and photodiodes were kept at the separation of $20 \mathrm{~mm}$ to record the signal from deeper tissue. Figure 3a shows the schematic of such probe having one OLED source and two photodiodes situated on a flexible base on the lower arm of a volunteer to detect the contribution of change in tissue oxygenation. The circular OLED source is based on $\mathrm{OC}_{1} \mathrm{C}_{10}$-PPV polymer, bought from OSRAM ${ }^{[18]}$ and two photodiodes were prepared using PTB7:PC ${ }_{70}$ BM as active layer. Normalized broad electroluminescence (EL) spectra of the OLED operated at a current of $80 \mathrm{~mA}$ with peak emission at $615 \mathrm{~nm}$ is shown in Figure 3b. The broad emission spectrum allowed the detection of different wavelength regions using filters on the detectors described in the methods section. For reference, the native unfiltered responsivity of the photodiodes, prepared by the blend of PTB7: $\mathrm{PC}_{70} \mathrm{BM}$ and measured at zero photodiode bias voltage, is also shown in the graph with a red line. The photodiode response without any filter exhibits a maximum at $690 \mathrm{~nm}$.

The performance of the sensor was demonstrated by inducing forearm ischemia. ${ }^{29,35]}$ A blood pressure cuff was applied to upper arm of a healthy volunteer who gave informed 
consent. The sensor was applied to the lower arm as shown in Figure 3a. The volunteer was kept at rest and in a stationary state with respect to blood pressure and heart rate for $10 \mathrm{~min}$. A blood pressure cuff on the upper arm was then inflated to $50 \mathrm{~mm} \mathrm{Hg}$ above systolic pressure for $3 \mathrm{~min}$. The inflated cuff shut off the blood supply to the forearm, which led to the change in the tissue oxyhemoglobin and is measured by recording the backscattered light from the sensor. Figure $3 \mathrm{c}$ shows the change in the tissue oxyhemoglobin absorption with time under different ischemic conditions for two different wavelengths at 610 and $700 \mathrm{~nm}$. It shows that sensor can measure the steady decrease in the tissue oxygenation during ischemia. When the cuff was released, change in signal overshot the baseline and then gradually returned to normal during relaxation period. The overshoot is a known physiological phenomenon. ${ }^{[29]}$ During ischemia, the forearm vessels respond to the deoxygenation by dilating, in order to increase the overall perfusion but are constrained by the total blood volume being kept constant by the occlusion. As a result, when the cuff is released more blood flows into the forearm than was initially present and the signal overshoots the initial baseline. The initial stationary value is recovered several minutes after release of the cuff.

As shown in Figure $3 \mathrm{c}$ the signal from both photodiodes reflects the decrease in tissue oxyhemoglobin during the ischemic period. The slope on the photodiode filtered at $700 \mathrm{~nm}$ is higher in comparison to the photodiode filtered at $610 \mathrm{~nm}$ because the difference in the absorption coefficient of $\mathrm{HbO}_{2}$ and $\mathrm{Hb}$ is larger at $700 \mathrm{~nm}$ than $610 \mathrm{~nm}$ (Figure S5, Supporting Information). And so results obtained at longer wavelength provide better information about tissue oxygenation. Since the optical signals were detected using a large separation of $20 \mathrm{~mm}$ between source and detector, the results are mainly from deep muscle tissue. The results show a time dependence of the backscattered light signals similar to those obtained by other setups employing more traditional combinations of inorganic lasers, ${ }^{[29]}$ LEDs, photodiodes, ${ }^{[35]}$ and photomultiplier tubes reported earlier. ${ }^{[34]}$

The organic optoelectronic sensors we have developed offer advantages in terms of performance, sensitivity, flexibility, and ease of fabrication over other sensors. For example, our muscle contraction sensor has advantages over the widely used $\mathrm{EMG}^{[36]}$ technique. The most important advantage is its ability to distinguish between isotonic and isometric muscle contractions. Our sensor provides more information regarding the mechanical state of the muscle than EMG, and can be used for finer control of prosthetic limbs in the motions for which isometric contractions are predominant, i.e., static holding of weights, pushing doors. ${ }^{[31]}$ Our sensor can also be used for compensating actuation of the prosthetics during various conditions for fine mimicking of gaits. ${ }^{[33]}$ A second advantage is that as it uses light it is non-invasive, avoiding the need for the needlelike electrodes used in EMG and complications coming with electrodes, such as stability to physiological body fluids during chronic measurements, biocompatibility, and local toxicity. The third advantage is it is flexible, thin, and compact so can conform to the shape the muscle in comparison to other optical muscle contraction techniques, such as diffuse correlation spectroscopy ${ }^{[37]}$ or electro-optical sensing using inorganic LED and photodiodes. ${ }^{[19]}$ The fourth advantage is that it is simple to make and had the prospect of being monolithic or made into arrays using inkjet printing techniques. Thus, our sensor has the potential to be useful for sustained monitoring of patients or rehabilitation. ${ }^{[3,38]}$

For the second sensor, several methods exist to access the tissue oxygenation, which has been widely studied in recent years for functional diagnosis and therapeutic monitoring of various diseases. For example, Doppler ultrasound measurements can probe deep tissue but are limited to large vessels. Magnetic resonance imaging and positron emission tomography are capable of monitoring deep tissue but require expensive and cumbersome equipment not available to all clinics or laboratories. ${ }^{[34]}$ The NIRS technique is non-invasive and can be used to make portable devices. But few commercial instruments using the NIRS technique are available to measure tissue oxygen metabolism. ${ }^{[39]}$ As shown by our proof of concept studies here, organic optoelectronics devices have advantages in terms of being thin, having low weight and high flexibility. Such sensors might also be useful for preclinical applications and open up the possibility of continuous monitoring of chronic diseases as well as for prosthetics.

In conclusion, we have demonstrated novel applications of organic optoelectronic devices in the field of wearable sensors for medicine with examples of a muscle contraction sensor and a tissue oxygenation sensor. The thin flexible muscle contraction sensor performance was tested for isometric and isotonic contractions. We demonstrated the application of this sensor by showing that it could be used to control a robotic arm that mimicked the movement of a real arm. We have also shown that an organic optoelectronic probe can provide a convenient way of measuring tissue oxygenation. With this technology the sensor components can be developed with solution processable methods, like inkjet printing or coating techniques on large areas or in multipixeled arrays, thus, significantly reducing size, complexity, and cost. This is combined with ease of wearing, making long-term monitoring during normal everyday activities possible. Our results provide a new direction for the application of organic optoelectronic devices in clinical medicine, rehabilitation, and sports.

\section{Experimental Section}

Participants: Informed consent was obtained from two participants who volunteered to perform these studies. All testing reported conformed to the ethical requirements of the University of St. Andrews and the declaration of Helsinki.

Preparation of Organic Devices: For the OPDs the polymer PTB7 was bought from 1-material company and $\mathrm{PC}_{70} \mathrm{BM}$ was bought from Solenne BV. The additive 1, 8-octanedithiol (DIO) was bought from Fluka. For OLEDs the organic active layer SY (PDY132) was bought from Merck-Covion. All the solvents used were bought from Sigma-Aldrich. The buffer layer poly $(3,4$ ethylenedioxythiophene) poly(styrenesulfonate) (PEDOT:PSS) was bought from Clevios and was used without further purification. The OLED source and OPDs were prepared on $12 \mathrm{~mm} \times 12 \mathrm{~mm}$ polyethylene terephthalate (PET) and glass substrates with indium tin oxide (ITO) top anode layer of $120 \mathrm{~nm}$. The substrates were carefully cleaned by ultrasound-assisted cleaning in water, acetone, and isopropyl alcohol for $15 \mathrm{~min}$. The organic layers on top of the anode were prepared 
by spin coating inside the glovebox with oxygen and water content less than $0.1 \mathrm{ppm}$. All the organic materials and solvents were used without further purification.

The OPD structure was: ITO-coated PET, a $40 \mathrm{~nm}$ layer of PEDOT:PSS by spin coating at $4000 \mathrm{rpm}$, a $90 \mathrm{~nm}$ layer of polymer PTB7 with $\mathrm{PC}_{70} \mathrm{BM}$ and $3 \% \mathrm{DIO}$ additive as active layer by spinning at $1000 \mathrm{rpm}$ followed by a layered metal cathode consisting of $20 \mathrm{~nm}$ calcium and $200 \mathrm{~nm}$ aluminum by using a thermal evaporator. The blend ratio of the polymer PTB7 and PC 70 BM was kept 1:1.5 with total concentration $25 \mathrm{mg} \mathrm{mL}^{-1}$ in the 1,4-dichlorobenzene solvent. Each photodiode consisted of a single pixel of dimensions $6 \mathrm{~mm} \times 6 \mathrm{~mm}$. The OLED structure was: ITO-coated PET, a $40 \mathrm{~nm}$ layer of PEDOT:PSS by spin coating at $4000 \mathrm{rpm}$, a $70 \mathrm{~nm}$ layer of polymer SY as an active layer by spinning at $1200 \mathrm{rpm}$ followed by a layered metal cathode consisting of $20 \mathrm{~nm}$ calcium and $200 \mathrm{~nm}$ aluminum by using a thermal evaporator. The SY polymer concentration was $5 \mathrm{mg} \mathrm{ml}^{-1}$ in chlorobenzene solvent. OLED source consisted of a single pixel of dimensions $3 \mathrm{~mm} \times 3 \mathrm{~mm}$. The active area of the photodiodes and OLED was encapsulated by PET film and which was attached using a UV curable epoxy (Norland 68) by shining $365 \mathrm{~nm}$ from low power UV lamp for $2 \mathrm{~min}$. The total thickness of the devices was less than $1 \mathrm{~mm}$. A $600 \mathrm{~nm}$ long-pass gelatin filter was used to select the red light emission from the broad light-emitting light source.

Fabrication of the Probe for Muscle Contraction Sensor: The design of the photomask to print the required circuit on plastic substrates was prepared by Rhino3D CAD software. The photomask was prepared by cutting Perspex Poly (methyl methacrylate) (PMMA) sheets of thickness $3 \mathrm{~mm}$ using a laser-cutting machine from CTR lasers UK. A $400 \mathrm{~nm}$ thick aluminum layer was deposited on the plastic substrates using a photomask inside the thermal evaporator. An OLED source and all four photodiodes were integrated on the printed circuit using silver conductive epoxy (Circuitworks CW2400) to provide the contacts to the devices. The thin copper wires were used to make contacts between the flexible probe head and the multicore cable, which connects the photodiodes and the OLED to the photodiode preamplifier and a continuous-wave OLED driver. The amplitude of the scattered light from muscle tissue is low, of less than $0.1 \%$ of that of the injected light so a preamplifier is needed to get the good signal to noise of the collected signal from the sensor. The schematic diagram of the amplifier for muscle contraction sensor with description is provided in Figure S6 (Supporting Information).

The complete sensor was tested with DENSO robotic arm (Model VP-6242G, DENSO Wave Inc., Japan) as a proof of concept for active prosthetic devices. The robotic arm consists of a single mechanical arm with an end effector at its tip. The signal collected by the probe is first sent through the amplifier and then converted to a digital signal by the I/O converter.

Fabrication of the Probe for Tissue Oxygen Sensor: The probe consists of two OPDs and an OLED source. The source is a circular OLED of $2 \mathrm{~cm}$ diameter based on the $\mathrm{OC}_{1} \mathrm{C}_{10}$-PPV polymer. The OPDs were prepared using PTB7 materials as explained above. Each photodiode consists of a single pixel of dimensions $6 \mathrm{~mm} \times 6 \mathrm{~mm}$. The responsivity of the photodiodes was measured using a monochromatic light source calibrated by a photodiode calibrated by the National Physical Laboratory of the UK. The photodiodes were rendered wavelength selective by using appropriate filters. In particular, for bandpass selectivity at $610 \mathrm{~nm}$ peak wavelength, a long-pass gelatin filter was used in combination with a filter prepared using cryptocyanine dye in a PMMA matrix, drop-cast from an ethanol solution, with total thickness of the filter around few $\mu \mathrm{m}$ only. Detection at $700 \mathrm{~nm}$ and beyond was achieved using a $700 \mathrm{~nm}$ acrylic long-pass filter. The thin filters were chosen because they reduce the asymmetrical distance between the source and the photodiodes and also help in reducing the stray light coming directly from the source to the photodiodes. The probe was designed on a flexible base and the separation between the source and the photodiodes was $20 \mathrm{~mm}$. Blackinsulated tape was applied to the edges of the devices to avoid direct stray light from the light source to the photodiodes. The signal from the photodiodes was measured by a multimeter (Keithley 2400) and was amplified by a custom transimpedance amplifier. Data were recorded using an analog-to-digital acquisition card controlled by a computer running a lab view program.

\section{Supporting Information}

Supporting Information is available from the Wiley Online Library or from the author.

\section{Acknowledgements}

This work was supported by Engineering and Physical Sciences Research Council Programme Grant "Challenging the limits of photonics: Structured light" (grant number EP/J01771X/1) and MRC. The authors are grateful to Mario E. Giardini for very helpful discussions and suggestions and use of the robot arm, and to Mark Ross and Christopher Booth for their assistance with the electronics.

Received: August 5, 2014

Revised: October 13, 2014

Published online:

[1] R. R. Holman, S. K. Paul, M. A. Bethel, H. A. W. Neil, D. R. Matthews, New Engl. J. Med. 2008, 359, 1565.

[2] P. Bonato, J. Neuroeng. Rehabil. 2005, 2, 2.

[3] C. N. Scanaill, S. Carew, P. Barralon, N. Noury, D. Lyons, G. M. Lyons, Ann. Biomed. Eng. 2006, 34, 547.

[4] T. Yamada, Y. Hayamizu, Y. Yamamoto, Y. Yomogida, A. Izadi-Najafabadi, D. N. Futaba, K. Hata, Nat. Nanotechnol. 2011, 6, 296.

[5] a) C. Pang, G.-Y. Lee, T.-i. Kim, S. M. Kim, H. N. Kim, S.-H. Ahn, K.-Y. Suh, Nat. Mater. 2012, 11, 795; b) M. K. Kwak, H.-E. Jeong, K. Y. Suh, Adv. Mater. 2011, 23, 3949.

[6] D.-H. Kim, N. Lu, R. Ma, Y.-S. Kim, R.-H. Kim, S. Wang, J. Wu, S. M. Won, H. Tao, A. Islam, K. J. Yu, T.-i. Kim, R. Chowdhury, M. Ying, L. Xu, M. Li, H.-J. Chung, H. Keum, M. McCormick, P. Liu, Y.-W. Zhang, F. G. Omenetto, Y. Huang, T. Coleman, J. A. Rogers, Science 2011, 333, 838.

[7] S. Gong, W. Schwalb, Y. Wang, Y. Chen, Y. Tang, J. Si, B. Shirinzadeh, W. Cheng, Nat. Commun. 2014, 5, 3132.

[8] J.-W. Jeong, W.-H. Yeo, A. Akhtar, J. J. S. Norton, Y.-J. Kwack, S. Li, S.-Y. Jung, Y. Su, W. Lee, J. Xia, H. Cheng, Y. Huang, W.-S. Choi, T. Bretl, J. A. Rogers, Adv. Mater. 2013, 25, 6839.

[9] S. R. Forrest, Nature 2004, 428, 911.

[10] M. Sessolo, D. Khodagholy, J. Rivnay, F. Maddalena, M. Gleyzes, E. Steidl, B. Buisson, G. G. Malliaras, Adv. Mater. 2013, 25, 2135.

[11] G. Li, R. Zhu, Y. Yang, Nat. Photonics 2012, 6, 153.

[12] a)J. Shinar, R. Shinar, J. Phys. D.: Appl. Phys. 2008, 41, 133001; b) G. Ryu, J. Huang, O. Hofmann, C. A. Walshe, J. Y. Y. Sze, G. D. McClean, A. Mosley, S. J. Rattle, J. C. deMello, A. J. deMello, D. D. C. Bradley, Lab Chip 2011, 11, 1664.

[13] O. Hofmann, X. H. Wang, J. C. deMello, D. D. C. Bradley, A. J. deMello, Lab Chip 2005, 5, 863.

[14] M. Ramuz, D. Leuenberger, L. Buergi, J. Polym. Sci., Part B: Polym. Phys. 2011, 49, 80.

[15] a) K. Takei, T. Takahashi, J. C. Ho, H. Ko, A. G. Gillies, P. W. Leu, R. S. Fearing, A. Javey, Nat. Mater. 2010, 9, 821; b) S. C. B. Mannsfeld, B. C. K. Tee, R. M. Stoltenberg, C. V. H. H. Chen, S. Barman, B. V. O. Muir, A. N. Sokolov, C. Reese, Z. Bao, Nat. Mater. 2010, 9, 859 .

[16] D. Ghezzi, M. R. Antognazza, M. Dal Maschio, E. Lanzarini, F. Benfenati, G. Lanzani, Nat. Commun. 2011, 2, 166. 
[17] D. Khodagholy, T. Doublet, P. Quilichini, M. Gurfinkel, P. Leleux, A. Chestem, E. Ismailova, T. Herve, S. Sanaur, C. Bernard, G. G. Malliaras, Nat. Commun. 2013, 4, 1575.

[18] S. K. Attili, A. Lesar, A. McNeill, M. Camacho-Lopez, H. Moseley, S. Ibbotson, I. D. W. Samuel, J. Ferguson, Brit. J. Dermatol. 2009, 161, 170.

[19] A. Chianura, M. E. Giardini, Med. Biol. Eng. Comput. 2010, 48, 731.

[20] T. Pohjolainen, H. Alaranta, M. Karkkainen, Prosthetics Orthotics Int. 1990, 14, 75.

[21] M. L. Schroeter, M. M. Bücheler, K. Müller, K. Uludağ, H. Obrig, G. Lohmann, M. Tittgemeyer, A. Villringer, D. Y. von Cramon, Neurolmage 2004, 21, 283.

[22] D. Robbins, C. Elwell, A. Jimenez, M. Goss-Sampson, J. Sports Sci. Med. 2012, 11, 346.

[23] M. Vardi, A. Nini, Eur. J. Vasc. Endovasc. 2008, 35, 68.

[24] M. V. Wolkenstein, Общая биосизика (General Biophysics), Vol. 5.2 [in Russian], Hayka (Nauka) Moscow, Russia, 1978.

[25] A. F. Huxley, R. Niedergerke, Nature 1954, 173, 971.

[26] Y. Liang, Z. Xu, J. Xia, S.-T. Tsai, Y. Wu, G. Li, C. Ray, L. Yu, Adv. Mater. 2010, 22, E135.

[27] S. Gambino, A. K. Bansal, I. D. W. Samuel, Org. Electron. 2013, 14, 1980.

[28] S. J. Matcher, C. E. Elwell, C. E. Cooper, M. Cope, D. T. Delpy, Anal. Biochem. 1995, 227, 54
[29] N. B. Hampson, C. A. Piantadosi, J. Appl. Physiol. 1988, 64, 2449.

[30] K. Matsushita, S. Homma, E. Okada, Proc SPIE Vol 3194: Photon Propagation in Tissues III, (Eds: D. A. Benaron, B. Chance, M. Ferrari, A. Katzir), SPIE, Bellingham, USA, 1998, 159.

[31] L. Bazzotti, Electromyogr. Clin. Neurophysiol. 1999, 39, 421.

[32] J. T. Gwin, D. P. Ferris, J. Neuroeng. Rehabil. 2012, 9.

[33] L. Juana, L. Weidab, H. Haiyanc, H. Sha, S. Lininge, presented at IEEE 7th International Conference on Nano/Molecular Medicine and Engineering (NANOMED 2013), Phuket, Thailand November 2013.

[34] M. Ferrari, M. Muthalib, V. Quaresima, Philos. Trans. R. Soc. A 2011, $369,4577$.

[35] M. E. Giardini, M. Corti, P. Lago, A. Gelmetti, Proc. SPIE Vol. 3911: Biomedical Diagnostic, Guidance, and Surgical-Assist Systems II, (Eds: T. Vo-Dinh, W. S. Grundfest, D. A. Benaron), SPIE, Bellingham, USA, 2000, 250.

[36] J. A. Kirchner, B. Wyke, Nature 1964, 203, 1243.

[37] M. Belau, M. Ninck, G. Hering, L. Spinelli, D. Contini, A. Torricelli, T. Gisler, J. Biomed. Opt. 2010, 15.

[38] H. S. Patwa, Nat. Clin. Pract. Neurol. 2009, 5, 78.

[39] M. Dujovny, J. I. Ausman, H. Stoddart, K. V. Slavin, G. D. Lewis, R. Widman, Neurosurgery 1995, 37, 160. 